\title{
Interspecific and host-related gene expression patterns in nematode-trapping fungi
}

\author{
Karl-Magnus Andersson ${ }^{1}$, Dharmendra Kumar ${ }^{2}$, Johan Bentzer ${ }^{1}$, Eva Friman ${ }^{1}$, Dag Ahrén ${ }^{3}$ and Anders Tunlid ${ }^{*}$
}

\begin{abstract}
Background: Nematode-trapping fungi are soil-living fungi that capture and kill nematodes using special hyphal structures called traps. They display a large diversity of trapping mechanisms and differ in their host preferences. To provide insights into the genetic basis for this variation, we compared the transcriptome expressed by three species of nematode-trapping fungi (Arthrobotrys oligospora, Monacrosporium cionopagum and Arthrobotrys dactyloides, which use adhesive nets, adhesive branches or constricting rings, respectively, to trap nematodes) during infection of two different plant-pathogenic nematode hosts (the root knot nematode Meloidogyne hapla and the sugar beet cyst nematode Heterodera schachtii).

Results: The divergence in gene expression between the fungi was significantly larger than that related to the nematode species being infected. Transcripts predicted to encode secreted proteins and proteins with unknown function (orphans) were overrepresented among the highly expressed transcripts in all fungi. Genes that were highly expressed in all fungi encoded endopeptidases, such as subtilisins and aspartic proteases; cell-surface proteins containing the carbohydrate-binding domain WSC; stress response proteins; membrane transporters; transcription factors; and transcripts containing the Ricin-B lectin domain. Differentially expressed transcripts among the fungal species encoded various lectins, such as the fungal fruit-body lectin and the D-mannose binding lectin; transcription factors; cell-signaling components; proteins containing a WSC domain; and proteins containing a DUF3129 domain. A small set of transcripts were differentially expressed in infections of different host nematodes, including peptidases, WSC domain proteins, tyrosinases, and small secreted proteins with unknown function.

Conclusions: This is the first study on the variation of infection-related gene expression patterns in nematode-trapping fungi infecting different host species. A better understanding of these patterns will facilitate the improvements of these fungi in biological control programs, by providing molecular markers for screening programs and candidates for genetic manipulations of virulence and host preferences.
\end{abstract}

Keywords: Comparative transcriptomics, Heterodera schachtii, Meloidogyne hapla, Nematode-trapping fungi

\section{Background}

Soil contains a diverse range of fungi that are parasites on nematodes [1]. These fungi include the nematode-trapping fungi, which have specific hyphal structures in which the nematodes can be trapped. The interest in studying these fungi is due to their potential use as biological control agents for plant and animal parasitic nematodes [2]. The traps of the nematode-trapping fungi develop from hyphae and can be formed spontaneously or be induced in response to signals from the environment [3]. There is a

\footnotetext{
* Correspondence: anders.tunlid@biol.lu.se

'Department of Biology, Microbial Ecology Group, Lund University, Ecology Building, 22362 Lund, Sweden

Full list of author information is available at the end of the article
}

large variation in the morphology of the traps, and the type of trap depends on the species [4]. In some species, the traps consist of an erect branch that is covered by an adhesive material. In other species such as in the well-studied Arthrobotrys oligospora, the trap is a three-dimensional net. A third type of trap is the adhesive knob, which is a single celled structure. Finally, there are some species that capture nematodes using a mechanical trap called a constricting ring [4]. Despite the large morphological variation in trapping structures, phylogenetic analyses inferred from molecular data has shown that the majority of the nematode-trapping fungi belong to a monophyletic group consisting of a single family of the order Orbiliales (Ascomycota) [5-8]. Furthermore, 
these studies have shown that the nematode-trapping fungi have evolved along two major lineages: one basal lineage leading to species with constricting rings and one lineage containing species that form adhesive traps, including three-dimensional networks, knobs and branches [5-8].

The trapping mechanisms of the species with constricting rings and those with adhesive traps are distinctively different [3]. The constricting ring consists of three cells. When the nematode enters the ring, the cells inflate and the nematode is trapped. The closure is very rapid $(0.1 \mathrm{~s})$ and is triggered by pressure of the nematode on the constricting-ring cells [9]. Ultrastructural examinations revealed that the cell wall of the constrictingring cells is folded; when the cells inflate, the folded cell wall balloons out and forms the new cell wall $[10,11]$. The adhesive trap is surrounded by a layer of fibrillar, extracellular polymers. Although the molecular mechanism has not yet been characterized, ultrastructural studies have shown that the fibrillar layer is reorganized during the attachment of the traps to the nematode cuticle [12]. Following the trapping of nematodes, the infection mechanisms appear to be rather similar in the species with constricting rings and adhesive traps: the fungus forms a penetration tube that pierces the nematode cuticle. During penetration the nematode becomes paralyzed. Subsequently, the internal tissues are rapidly colonized and digested by fungal hyphae [13].

In laboratory assays, most nematode-trapping fungi can trap and infect a range of different nematode species $[14,15]$. However, there are a number of studies showing that different species and even strains of nematode-trapping fungi can vary in their host preferences. For example, in vitro predacity tests of four nematode-trapping fungi showed that the constricting-ring species Arthrobotrys dactyloides was the most efficient species in capturing and killing the root knot nematode Meloidogyne graminicola [16]. Further studies on A. dactyloides showed that even strains of this species differed in their predacity to Meloidogyne incognita [17]. Significant differences in the susceptibility to nematode-trapping fungi have also been shown in field trials with the cyst nematode Heterodera schachtii and the root knot nematode Meloidogyne javanica [18].

Recently, the infection mechanism of nematode-trapping fungi has been examined using the tools of genomics, transcriptomics and proteomics. The genomes of two nematode-trapping fungi have been published; the net-forming Arthrobotrys oligospora [19] and the adhesive knob-forming Monacrosporium haptotylum [20]. The two genomes are similar in size and consist of $\sim 62 \%$ core genes that are shared with other fungi, $\sim 20 \%$ genes that are specific for the two species and $\sim 16 \%$ genes that are unique for each genome [20]. Comparative genome analysis showed that the genomes of nematode-trapping fungi have been expanded in a number of gene families, including extracellular peptidases such subtilisins; homologs to several virulence factors identified in plant-pathogenic fungi; and families of putative cell-surface adhesins containing carbohydrate-binding domains such as the WSC domain and the mucin domain [20]. Transcriptome analysis showed that $M$. haptotylum expresses a unique set of genes during the early stages of infection of the nematode Caenorhabditis briggsae. Among these is a large proportion that belongs to gene families that are significantly expanded in the nematode-trapping fungi. Transcripts encoding small secreted proteins (SSPs) and many species-specific genes were also highly expressed during the early phase of infection. Many of them were orphans, that is, genes lacking both homologs and Pfam domains [20,21]. Furthermore, quantitative proteomics revealed proteins that were significant upregulated in the knob compared with the vegetative mycelia in M. haptotylum. Among the upregulated proteins were peptidases, tyrosinase and proteins containing the WSC domain [22].

In this study, we have examined in more detail the molecular basis of the infection process in nematodetrapping fungi that have various trapping mechanisms, including adhesive nets (A. oligospora), adhesive branches (Monacrosporium cionopagum) and constricting rings (A. dactyloides) $[4,13]$. Two plant-parasitic nematodes were used as hosts, the root knot nematode Meloidogyne hapla and the sugar beet cyst nematode $H$. schachtii. These are both sedentary endoparasites belonging to the group of nematodes that causes the most damages to crops [23]. Comparative transcriptome analysis of the infection, including the adhesion, penetration and digestion stages, showed that the divergence in interspecific gene expression was significantly larger than that related to the nematode host used. We identified a common set of genes that were expressed by all three fungi and a more variable set that were regulated depending on either the fungal species or the nematode host.

\section{Results and discussion}

\section{Infection experiments}

The nematodes $M$. hapla or $H$. schachtii were added to plates containing the nematode-trapping fungi $A$. oligospora, $M$. cionopagum or $A$. dactyloides and the infection was followed under a light microscope (Figure 1). The following five combinations of fungi and nematodes were examined: A. oligospora and M. hapla (designated Ao(Mh)), A. oligospora and $H$. schachtii $(\mathrm{Ao}(\mathrm{Hs})), A$. dactyloides and M. hapla ( $\operatorname{Ad}(\mathrm{Mh})), A$. dactyloides and H. schachtii (Ad $(\mathrm{Hs})$ ), and $M$. cionopagum and $H$. schachtii $(\mathrm{Mc}(\mathrm{Hs}))$. There was a large difference in the infection rate, both between the nematodes and between the fungi (Table 1). Cyst nematodes (H. schachtii) showed lower susceptibility to nematode-trapping fungi than did root knot nematodes 


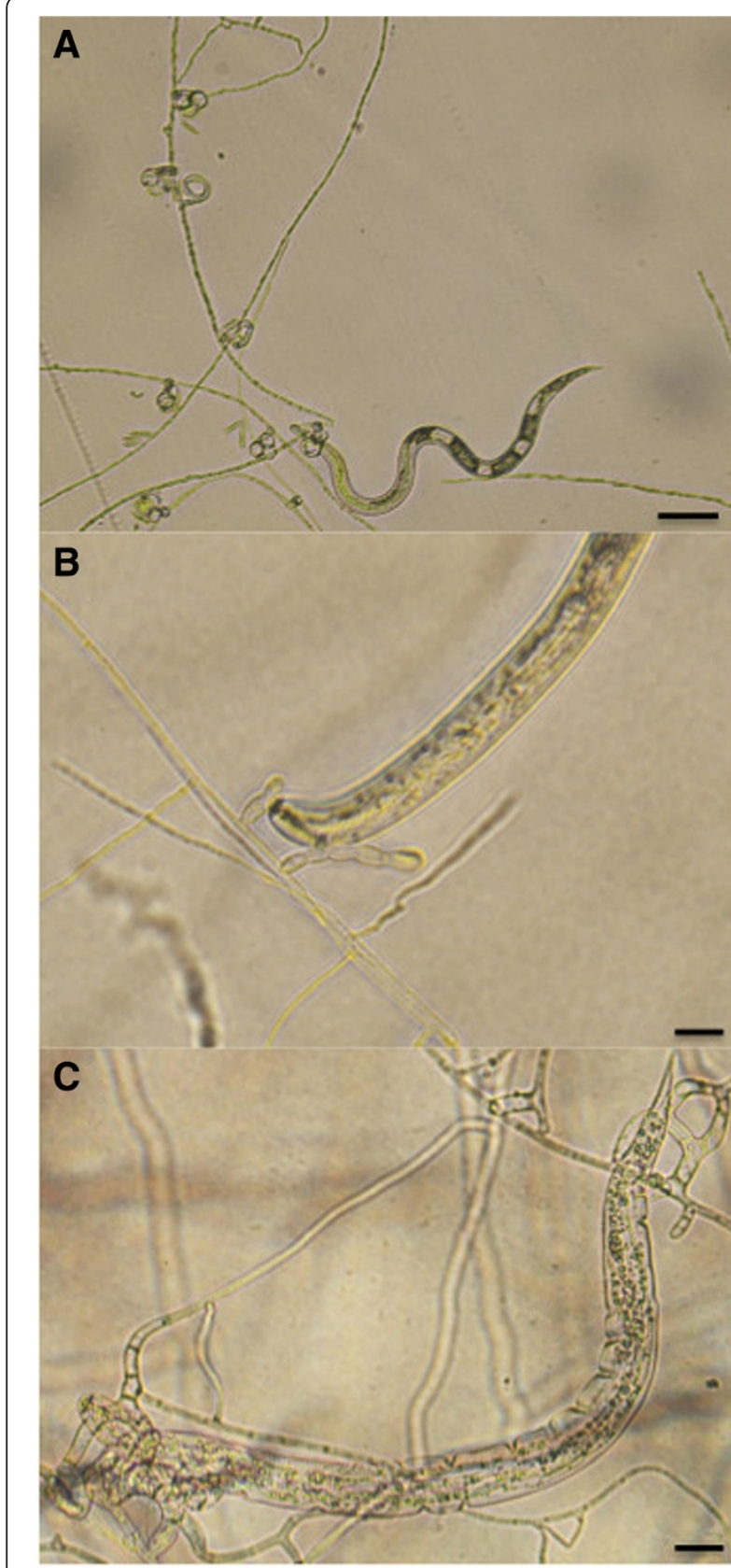

Figure 1 Micrographs of fungal and nematode interactions.

(A) Arthrobotrys dactyloides (constricting rings) after trapping the root knot nematode Meloidogyne hapla (bar $=50 \mu \mathrm{m}$ ). (B) Monacrosporium cionopagum (adhesive branches) after trapping and immobilizing the sugar beet cyst nematode Heterodera schachtii (bar $=20 \mu \mathrm{m})$. (C) Arthrobotrys oligospora (adhesive nets) after trapping, immobilization and colonization of H. schachtii (bar $=20 \mu \mathrm{m})$.

(M. hapla), which is in agreement with an earlier study by Jaffee and Muldoon [18]. This might be due to differences in the composition of the nematode cuticle [24]. Between the fungi, $A$. dactyloides infected $M$. hapla at a faster rate than A. oligospora infected M. hapla. A. dactyloides
Table 1 Infection of plant parasitic nematodes by nematode-trapping fungi ${ }^{a}$

\begin{tabular}{|c|c|c|c|c|c|}
\hline & $\mathrm{Ad}(\mathrm{Mh})$ & Ao(Mh) & $\mathrm{Ad}(\mathrm{Hs})$ & Ao(Hs) & $\mathrm{Mc}(\mathrm{Hs})$ \\
\hline \multicolumn{6}{|c|}{ Trapped } \\
\hline $3 \mathrm{~h}$ & $80.3(3.8)$ & & & & \\
\hline $7 \mathrm{~h}$ & & $40.5(2.6)$ & & & \\
\hline $12 \mathrm{~h}$ & & & $30.3(3.7)$ & $50.0(3.3)$ & $50.6(2.5)$ \\
\hline \multicolumn{6}{|c|}{ Paralyzed } \\
\hline $12 \mathrm{~h}$ & $90.8(2.5)$ & $30.4(2.4)$ & & & \\
\hline $24 \mathrm{~h}$ & & & $31.0(2.4)$ & & \\
\hline $32 \mathrm{~h}$ & & & & $50.7(2.9)$ & \\
\hline $36 \mathrm{~h}$ & & & & & $60.7(4.1)$ \\
\hline \multicolumn{6}{|c|}{ Colonized } \\
\hline $20 \mathrm{~h}$ & $92.7(3.2)$ & & & & \\
\hline $24 \mathrm{~h}$ & & $64.7(2.3)$ & & & \\
\hline $36 \mathrm{~h}$ & & & $35.3(3.0)$ & & \\
\hline $40 \mathrm{~h}$ & & & & $60.3(2.7)$ & \\
\hline $48 \mathrm{~h}$ & & & & & $55.2(2.4)$ \\
\hline
\end{tabular}

${ }^{a}$ Shown is the percentage (mean (SD, $\left.n=10\right)$ ) of the added nematodes that were trapped, paralyzed and colonized. Ao(Mh) denotes $A$. oligospora infecting M. hapla; $\mathrm{Ao}(\mathrm{Hs})$, A. oligospora infecting H. schachtii; $\mathrm{Ad}(\mathrm{Mh})$, A. dactyloides infecting M. hapla; $\mathrm{Ad}(\mathrm{Hs})$, A. dactyloides infecting H. schachtii; and $\mathrm{Mc}(\mathrm{Hs})$, $M$. cionopagum infecting $H$. schachtii.

colonized $H$. schachtii at a faster rate than the other species, especially $M$. cionopagum. The observation that species with constricting rings are more efficient in capturing and killing nematodes than nematode-trapping fungi with other types of trapping structures has been made in earlier studies [16].

\section{Characterization of the transcriptome libraries}

The number of reads obtained by the 454 sequencing of the five cDNA libraries corresponding to these species combinations ranged from 70061 to 245264 (Table 2). Based on these sequences, three different data sets were created (Figure 2). To generate the "Highly expressed transcripts" data set, the reads were assembled into isotigs (transcripts), and low abundance reads ( $<5$ reads), short isotigs $(<100$ base pairs, bp) and non-fungal sequences were removed. The number of the filtered isotigs in the five libraries varied between 1318 and 5140 and their average sizes varied between 1008 to $1237 \mathrm{bp}$. Almost all of the assembled isotigs (98.9\%) had fungal matches: a few matched species from Nematoda $(0.9 \%)$ and a few matched other species $(0.2 \%)$. To identify the set of transcripts that was most highly expressed in each library, the reads of the isotigs were normalized using the reads per kilobase pair method. This method fitted better than the reads per kilobase per million read (RPKM) method to the expectation that most transcripts have similar relative expression abundance between samples (Additional file 1). In each 
Table 2 Characterization of the transcriptome libraries

\begin{tabular}{|c|c|c|c|c|c|}
\hline Library $^{a}$ & Ao(Mh) & Ao(Hs) & $\operatorname{Ad}(\mathrm{Mh})$ & $\operatorname{Ad}(\mathrm{Hs})$ & $\mathrm{Mc}(\mathrm{Hs})$ \\
\hline \multicolumn{6}{|l|}{ Reads } \\
\hline Total number of reads & 114418 & 70061 & 226301 & 191632 & 245264 \\
\hline Filtered reads ${ }^{\mathrm{b}}$ & 97770 & 58208 & 183433 & 141506 & 206854 \\
\hline \multicolumn{6}{|l|}{ Isotigs } \\
\hline Total number of isotigs & 2663 & 1354 & 4517 & 4003 & 5258 \\
\hline Fungal isotigs & 2514 & 1261 & 4021 & 3560 & 4741 \\
\hline Nematode isotigs & 11 & 20 & 21 & 18 & 44 \\
\hline Others & 0 & 5 & 8 & 4 & 16 \\
\hline Filtered isotigs ${ }^{c}$ & 2634 & 1318 & 4428 & 3926 & 5140 \\
\hline (Average size, bp) & $\left(\begin{array}{ll}1 & 160\end{array}\right)$ & $(1008)$ & (1 237) & $\left(\begin{array}{ll}1 & 128\end{array}\right)$ & $\left(\begin{array}{ll}1 & 167\end{array}\right)$ \\
\hline Isotigs with Pfam & 1730 & 856 & 2317 & 2504 & 3071 \\
\hline Number of unique Pfam & 1251 & 707 & 1555 & 1592 & 1847 \\
\hline Isotigs with UniRef50 & 2485 & 1239 & 3916 & 3472 & 4601 \\
\hline Unique UniRef50 & 2326 & 1174 & 3101 & 3230 & 3981 \\
\hline \multicolumn{6}{|l|}{ Mapping to Ao genome } \\
\hline Number of mapped reads & 73623 & 40051 & & & \\
\hline Number of gene models ${ }^{d}$ & 7351 & 6377 & & & \\
\hline
\end{tabular}

${ }^{\mathrm{a}}$ The following five combinations of fungi and nematodes were characterized: A. oligospora and M. hapla (Ao(Mh)), A. oligospora and H. schachtii (Ao(Hs)), A. dactyloides and M. hapla $(\mathrm{Ad}(\mathrm{Mh}))$, A. dactyloides and H. schachtii $(\mathrm{Ad}(\mathrm{Hs}))$, and M. cionopagum and H. schachtii $(\mathrm{Mc}(\mathrm{Hs}))$.

${ }^{\mathrm{b}}$ Number of reads after removal of rRNA sequences.

${ }^{\mathrm{C}}$ Number of isotigs after removal of non-fungal sequences, low abundance isotigs (less than 5 reads), and short isotigs (<100 bp).

${ }^{\mathrm{d}}$ Number of gene models that have $\geq 1$ read.

library, the 500 most expressed isotigs (the "Top 500 transcripts") were analyzed.

Comparing transcriptomes between species with not yet sequenced genomes is challenging due to the difficulties in identifying one-to-one orthologs. To circumvent this problem, we here clustered the isotigs into UniRef50 clusters. UniRef50 clusters are based on pre-computed sequence clusters of the UniProt database that have at least 50 percent similarity and 80 percent coverage [26]. Istotigs were only grouped to UniRef50 clusters if passing a given threshold value (1e-10) and only one isotig per fungal species (displaying the highest sequence similarity) were assigned to a given UniRef50 cluster. A recent study including data from seven fungal genomes revealed that the grouping of gene sequences into UniRef50 clusters using the described procedure are in close agreement with traditional ortholog clustering methods (Canbäck et al., manuscript in preparation). Furthermore, the risk of clustering non-orthologous gene duplicates into a given UniRef50 cluster is reduced in nematode-trapping due to the rapid divergence of gene duplicates generated by repeat induced point (RIP) mutations [19,20]. In our analyses, 15713 of the in total 17446 isotigs matched to 6 520 unique UniRef50 protein clusters. The reads of these putative orthologs were normalized with DESeq [27]. Based on the hypothesis that most transcripts are not differentially expressed, the analysis showed that a proper normalization was obtained using the $5 \%$ most highly expressed UniRef50 clusters (Additional file 2). This cohort ("Differentially expressed UniRef50 clusters") contained 326 unique UniRef50 clusters.

The third data set, "Host-specific gene expression", was generated for identifying genes that were differentially expressed due to the nematode host species. Because the genome of A. oligospora is available [19], the analysis focused on comparing the transcriptional response of this fungus when infecting $M$. hapla and $H$. schachtii. The reads from the $\mathrm{Ao}(\mathrm{Mh})$ library were mapped to 7351 genes and those from the Ao(Hs) library to 6377 genes (Table 2).

\section{Divergence in gene expression}

To compare the functional groups of genes that were expressed in the different libraries, the abundances and expression levels of Pfam domains in the "Top 500 transcripts" data set were analyzed. The number of Pfam domains found in the five libraries varied between 330 and 412 (Additional files 3 and 4). In total, 700 Pfam domains were found in at least one of the libraries.

A principal component analysis (PCA) of the abundances of the Pfam domains showed that the libraries mainly clustered according to the fungal species (Figure 3A). The first axis (explaining $37 \%$ of the variability) separated the two libraries of $A$. dactyloides $(\mathrm{Ad}(\mathrm{Mh})$ and $\mathrm{Ad}(\mathrm{Hs}))$ from those of $A$. oligospora ( $\mathrm{Ao}(\mathrm{Mh})$ and $\mathrm{Ao}(\mathrm{Hs}))$. The 


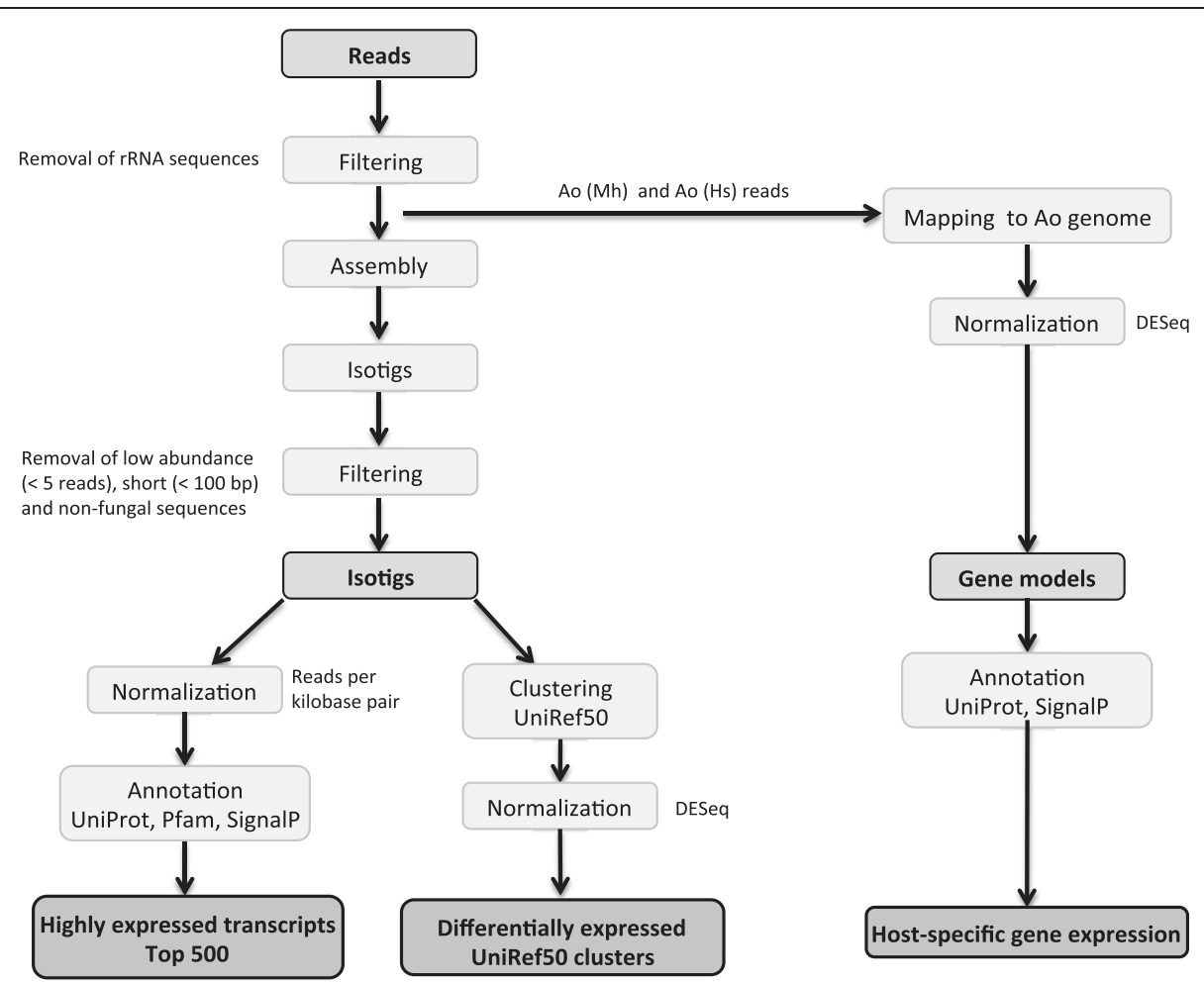

Figure 2 Flowchart of the data analysis. Five different cDNA libraries were sequenced and the reads were used to generate three different data sets. First, the data set "Highly expressed transcripts" were retrieved by assembling the reads of each library into isotigs (ranscripts) and normalizing the read counts. Using this approach, the 500 most highly expressed transcripts in each library were retrieved (the "Top 500" data set). Second, the data set "Differentially expressed UniRef50 clusters" was obtained by matching the isotig sequences using BLASTX [25] to UniRef50 clusters [26]. The procedure organized the isotigs into putative orthologs for which expression levels could directly be compared between the five libraries. Third, to identify the data set "Host-specific gene expression" the reads from the two libraries of A. oligospora were mapped to the genome sequence of this fungus [19]. Ao(Mh) denotes A. oligospora and M. hapla; Ao(Hs), A. oligospora and H. schachtii; Ad(Mh), A. dactyloides and M. hapla; Ad $(\mathrm{Hs})$, A. dactyloides and H. schachtii; and Mc(Hs), M. cionopagum and H. schachtii. Further details of the libraries are shown in Table 2.

second axis $(27 \%)$ separated $M$. cionopagum $(\mathrm{Mc}(\mathrm{Hs}))$ from the two Arthrobotrys species. Clearly, in both A. dactyloides and A. oligospora, the divergence in Pfam expression patterns associated with fungal species was larger than that related to the host nematode species.

Analysis of the expression levels of the UniRef50 clusters confirmed these patterns. A PCA based on the 5\% most highly expressed UniRef50 clusters showed that the first axis (49\%) separated the two libraries of $A$. dactyloides from the two libraries of A. oligospora (Additional file 5). M. cionopagum was separated from the other libraries along the second axis (26\%). Scatter plots showed that the numbers of UniRef50 clusters that were differentially expressed more than twofold between the fungal species were greater than between the host nematode species (Figure 4).

\section{Commonly expressed transcripts}

In agreement with a previous study examining the infectionregulated transcriptome of $M$. haptotylum [20], the highly expressed transcripts of $A$. dactyloides, A. oligospora and $M$. cionopagum were enriched with those predicted to encode proteins with a secretion signal and those encoding orphans (Table 3). To characterize the core set of transcripts that was highly expressed by all fungi, the commonly expressed Pfam domains of $A$. dactyloides, A. oligospora and $M$. cionopagum were identified (Figure 3B). In total this set contained 206 Pfam domains that were annotated into putative functions (Table 4; Additional file 6). The core set of Pfam domains included several protein families that have previously been identified to be highly expressed by A. oligospora and $M$. haptotylum during infection of $C$. briggsae, such as subtilisin (peptidase_S8), aspartyl peptidases, CFEM (a fungal specific cysteine-rich domain that is found in some proteins with proposed roles in fungal pathogenesis) [28], and the carbohydrate-binding WSC domain [20,22]. In addition, the core set contained a number of Pfam domains found in proteins involved in fungal stress response, cell signaling, organization of the cytoskeleton, vesicular transport and membrane transport, as well as several families of calcium-binding proteins and transcription factors. Domains of enzymes and proteins involved in the carbon, energy and amino-acid metabolism and protein synthesis were also highly expressed. 

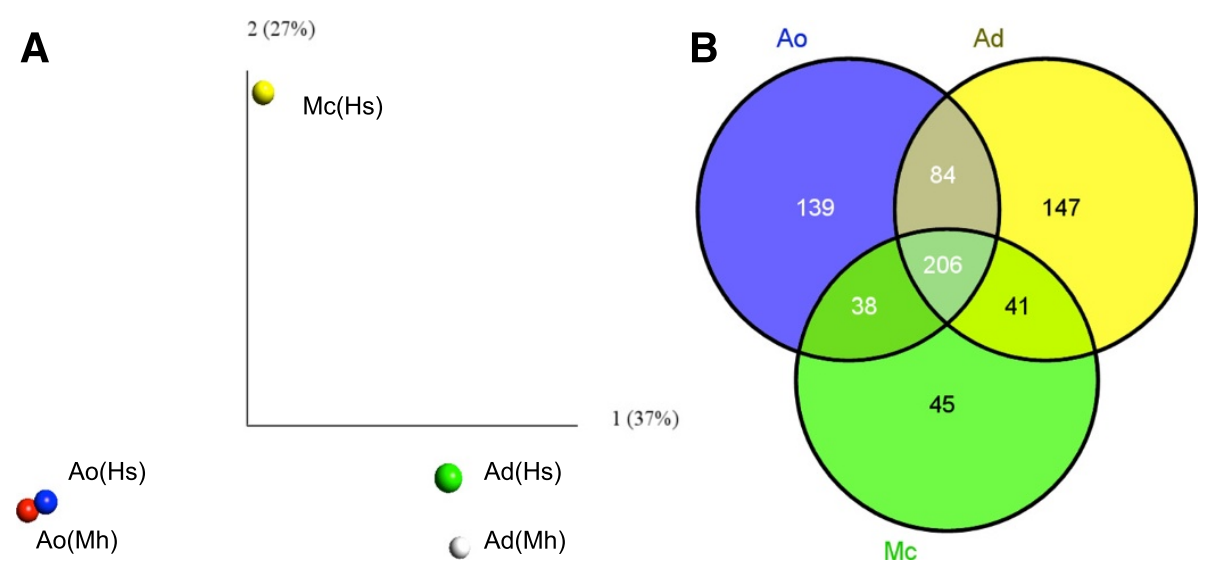

Figure 3 Expression patterns of Pfam domains among the most highly expressed transcripts. (A) Principal component analysis (PCA) of the abundance of Pfam domains. Each point in the PCA plot corresponds to a CDNA library. Ao(Mh) denotes A. oligospora and M. hapla; Ao(Hs), A. oligospora and H. schachtii; Ad(Mh), A. dactyloides and M. hapla; Ad(Hs), A. dactyloides and H. schachtii; and Mc(Hs), M. cionopagum and H. schachtii. The PCA was performed on the read counts of 700 Pfam domains $\left(\log _{2}\right.$ transformed $($ counts +1$)$ ). (B) Venn diagram of highly expressed Pfam domains. Shown is the distribution of the in total 700 unique Pfam domains that were found among the top 500 most expressed transcripts in A. oligospora (Ao), A. dactyloides (Ad) and M. cionopagum (Mc). 'Ao' contains all domains that were found among the 500 most expressed transcript in one or in both of the $\mathrm{Ao}(\mathrm{Mh})$ and $\mathrm{Ao}(\mathrm{Hs})$ libraries. 'Ad' contains all domains that were found among the 500 most expressed transcripts in one or in both of the $\mathrm{Ad}(\mathrm{Mh})$ and $\mathrm{Ad}(\mathrm{Hs})$ libraries. 'Mc' contains all domains that were found among the 500 most expressed transcript in $\mathrm{Mc}(\mathrm{Hs})$.

Furthermore, transcripts containing the Atg8 domain were highly expressed in all fungi. Atg8 is an essential protein in the autophagic pathway [29] and disruption of a homolog of this gene in A. oligospora leads to reduced trap formation [30]. In addition, all three fungi expressed transcripts with the Pfam domain RicinB_lectin_2 (PF14200). Ricin-B lectins are ribosome-inactivating proteins (RIPs) consisting of a catalytic A-chain and a sugar-binding B-chain [31,32]. All fungi have a RicinB_lectin_2 transcript that match to G1X3G7 in A. oligospora. G1X3G7 is a protein with a length of 134 amino-acid residues (aa), without a secretion signal and with low sequence similarity to other RicinB lectins in the UniProt database. A RicinB_lectin_2 domain-containing protein (MOA) with nematotoxic activity against Caenorhabditis elegans has been identified in the basidiomycete Marasmius oreades [33]. The nematotoxicity was dependant on the cysteine protease activity of MOA and the binding of its lectin domain to glycosphingolipids in the worm intestine. MOA consists of 293 aa and lacks a classical secretion signal [33]. Sclerotinia sclerotiorum agglutinin (SSA) is a RicinB_lectin_2 domain-containing protein with a length more similar to the G1X3G7 protein in A. oligospora [34]. SSA has a length of 153 aa, lacks secretion signal and shows insecticidal properties when fed to the pea aphid Acyrthosiphon pisum [34].

Previous studies have shown that subtilisins (peptidase_S8) are important virulence factors in nematode-trapping fungi. In $A$. oligospora they have a key role in the early stages of infection, including immobilization of the captured nematode $[19,35,36]$. A. oligospora has 52 genes containing the peptidase_S8 domain [20]. However, only one transcript containing the peptidase_S8 domain was identified among the highly expressed transcripts in each library of Ao(Mh) and $\mathrm{Ao}(\mathrm{Hs})$. BLASTX searches showed that both transcripts displayed the highest sequence homology to the $A$. oligospora protein G1XLL2. The other cDNA libraries contained also only one transcript with the peptidase_S8 domain among the top 500 expressed genes. The three transcripts in $\mathrm{Ad}(\mathrm{Mh}), \mathrm{Ad}(\mathrm{Hs})$ and $\mathrm{Mc}(\mathrm{Hs})$ all displayed the highest sequence homology to $\mathrm{H} 072 \_8474$ in $M$. haptotylum. Interestingly, G1XLL2 and H072_8474 are orthologs (T. Meerupati, B. Canbäck, D. Ahrén, A. Tunlid, manuscript in preparation). Furthermore, G1XLL2 was the most expressed peptidase_S8 gene in A. oligospora during early infection ( 6 and 10 hours) of C. briggsae, and H072_8474 was the second most expressed peptidase_S8 gene in $M$. haptotylum during early infection (4 hours) of C. briggsae [20]. H072_8474 was also identified in the proteome of both the knob and the mycelia in $M$. haptotylum [22]. This shows that despite the large number of peptidase_S8 genes only a few are highly expressed during infection.

Stress proteins were highly expressed in all fungi independent of trapping structure. They included heatshock proteins and chaperones such as DnaJ, HSP70 and HSP90; gluthatione S-transferases; and antioxidant enzymes such as thioredoxin and catalase. Antioxidants are enzymes involved in the protection of the cell from oxidative damages induced by reactive oxygen species (ROS) [37]. ROS are continuously produced in the cell as byproducts from various metabolic pathways and have an 

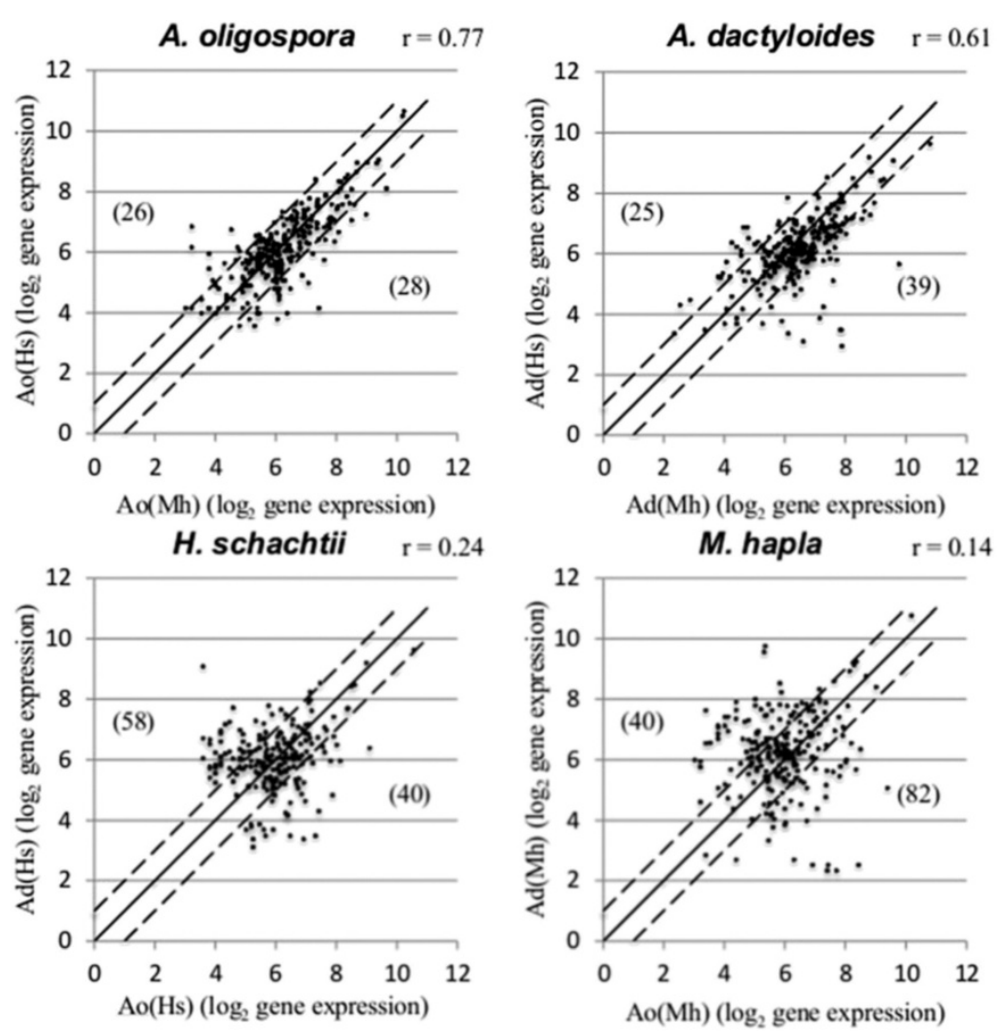

Figure 4 Expression levels of highly expressed UniRef50 clusters. $\log _{2}$ scatter plot of gene expression pattern between A. oligospora and A. dactyloides infecting different nematodes (top) and H. schachtii and M. hapla infected by different fungi (bottom). The gene expression levels (normalized, $\log _{2}$-transformed read counts) of the $5 \%$ most highly expressed UniRef50 proteins represented by 326 unique IDs are shown. The Pearson correlation coefficients $(r)$ of the comparisons are also shown. The diagonal line $(y=x)$ shows UniRef50 clusters with nearly identical expression levels. The dotted lines correspond to a twofold expression difference. The numbers of clusters that differ in expression level more than twofold are shown in parentheses.

important role(s) in signaling [38]. In the plant-pathogenic fungus Magnaporthe grisea, ROS-generating NADPH oxidases (Nox1 and Nox2) are essential for pathogenicity [39]. The authors [39] suggest that the generated ROS accumulate in the appressorium to facilitate oxidative crosslinking of cell-wall proteins. This leads to a strengthening of the cell wall of the appressorium that will eventually resist high turgor pressure [39]. Transcripts with sequence similarity to the Nox proteins in M. grisea were regulated in all fungi of our study during infection. However, none of them were found among our top 500 most expressed transcripts.

Table 3 Proportion (\%) of secreted proteins and orphans ${ }^{a}$

\begin{tabular}{|c|c|c|c|c|c|}
\hline Category & Ao(Mh) & Ao(Hs) & $\operatorname{Ad}(\mathrm{Mh})$ & $\mathrm{Ad}(\mathrm{Hs})$ & $\mathrm{Mc}(\mathrm{Hs})$ \\
\hline \multicolumn{6}{|l|}{ Secreted proteins } \\
\hline All isotigs & 8.4 & 9.4 & 7.8 & 8.6 & 9.1 \\
\hline Top 500 most expressed isotigs & 12.2 & 12.6 & 12.6 & 10.8 & 13.8 \\
\hline$\left(P^{\mathrm{b}}\right)$ & $(0.00031)$ & $(0.00070)$ & $(0.00002)$ & $(0.01205)$ & $(0.00008)$ \\
\hline \multicolumn{6}{|l|}{ Orphans ${ }^{c}$} \\
\hline All isotigs & 6.6 & 7.1 & 8.1 & 7.5 & 6.9 \\
\hline Top 500 most expressed isotigs & 7.8 & 7.6 & 12.8 & 9.6 & 9.2 \\
\hline$\left(P^{\mathrm{b}}\right)$ & $(0.03792)$ & $(0.07609)$ & $(0.00004)$ & $(0.01176)$ & $(0.00818)$ \\
\hline
\end{tabular}

aproportion of secreted proteins and orphans among all identified isotigs (i.e. transcripts) and among the 500 most expressed isotigs in each sample. The samples are: A. oligospora infecting M. hapla (Ao(Mh)), A. oligospora infecting H. schachtii $(\mathrm{Ao}(\mathrm{Hs}))$, A. dactyloides infecting M. hapla (Ad(Mh)), A. dactyloides infecting H. schachtii $(\mathrm{Ad}(\mathrm{Hs}))$, and $M$. cionopagum infecting $H$. schachtii $(\mathrm{Mc}(\mathrm{Hs}))$.

${ }^{\mathrm{b}}$ The probability $(P)$ of observing a given number of isotigs within the functional category by chance using the hypergeometric distribution.

'Isotigs that lack known homologs and do not contain any Pfam domains. 
Table 4 Pfam domains expressed by all fungi during nematode infection ${ }^{a}$

\begin{tabular}{ll}
\hline Putative functions & Pfam domains \\
\hline Peptidase & Peptidase_S8 (PF00082); Aspartyl protease (PF00026); Peptidase_M3 (PF01432); Peptidase_S10 (PF00450); \\
$\begin{array}{l}\text { Cell-surface proteins } \\
\text { Others }\end{array}$ & WSC (PF01822) \\
Stress response, & CFEM (PF05730); Ricin-type lectin (PF14200) \\
chaperons & Thioredoxin (PF00085); Glutathione S-transferase (PF00043); Catalase (PF00199); AhpC/TSA family (PF00578); DnaJ \\
Cell signaling & RHO protein GDP dissociation inhibitor (PF02115); ADP Ribosylation Factors (ARFs) (PF00025); 14-3-3- proteins (PF00244); \\
& Pkinase (PF00069); Ras (PF00071) \\
Calcium-binding & Calreticulin (PF00262); EF-hand motif (PF13499) \\
protein & \\
Cytoskeleton & Actin (PF00022); Cofilin (PF00241); Profilin (PF00235); Tropomyosin (PF12718); Tubulin (PF00091) \\
Autophagy & Atg8 (PF02991) \\
Peroxisome & Membrane protein Mpv17_PMP22 (PF04117) \\
Trancription & Multiprotein bridging factor 1 (PF08523); Homeobox (PF00046); TATA binding protein (PF00352); bZIP Transcription \\
& factor (PF00170, PF07716); Histone (PF00125, PF00538); Nucleosome assembly protein (PF00956); Helicases (PF00270,
\end{tabular}

Membrane transport $\quad$ ABC transporter (PF00005) Amino acids permease (PF00324); Ammonium transporter (PF00909); Major Facilitator Superfamily (PF07690); Sugar and others (PF00083); Porin (PF01459)

Vesicular transport Syntaxin (PF05739); Synaptobrevin (PF00957); Rab GDP dissociation inhibitors (PF00996)

Metabolism, glycolysis/ Enolase (PF03952); Fructose-bisphosphate aldolase (PF01116); Triose-phosphate isomerase (PF00121); Glyceraldehyde gluconeogenesis 3-phosphate dehydrogenase (PF00044); Phosphoglycerate kinase (PF00162); Pyruvate kinase (PF00224); Pyruvate carboxylase (PF00682)

Metabolism, pentose Phosphogluconate dehydrogenase (PF03446, PF00479); Transaldolase (PF00923); Transketolase (PF00456) phosphate pathway

Metabolism, TCA lactate/malate dehydrogenase (PF02866, PF00056); Succinyl coenzyme A synthetase (PF00549)

Metabolism, energy Cyt-b5 (PF00173); ATP synthase (PF00887, PF00137); mitochondrial carrier (PF00153); ATPases (PF00006, PF02874)

Metabolism, amino Glutamine amidotransferases (PF00310); NAD-specific glutamate dehydrogenase (PF10712); Glutamine synthetase (PF03951)

acids

Metabolism,

miscellaneous

Glycoside hydrolase family 1 (PF00232); Biotin-requiring enzyme (PF00364) aldo-keto reductase family (PF00248); Aldehyde dehydrogenase family (PF00171); Short-chain dehydrogenases/reductases family (PF00106); Pyrophosphatase (PF00719); Myo-inositol-1-phosphate synthase(PF01658, PF07994); FA desaturase (PF00487); Nucleoside diphosphate kinase (PF00334); UDP-glucose pyrophosphorylase (PF01704); Transketolase, pyrimidine binding domain (PF02779); ATPase (PF00004); CoA binding domain (PF02629) ATP-grasp_2 (PF08442); Calcineurin-like phosphoesterase (PF00149); Epimerase (PF01370)

Protein degradation Proteasome (PF00227, PF10584); Ubiquitination (PF00240, PF00240, PF00179)

Protein synthesis Elongation factors (PF10587, PF01873, PF00009); tRNA synthetases (PF00587); translation, initiation factors W2 (PF02020), SUI1 (PF01253), MIF4G (PF02854); Ribosomal proteins (PF00428, PF00428, PF00687, PF00466, PF00238, PF00827, PF00252, PF14204, PF01775,PF00828, PF00861, PF01280, PF03947, PF01157, PF01776, PF01246, PF01777, PF00831, PF00297, PF01 198, PF01655, PF01780, PF01907, PF01020, PF00935, PF00281, PF00347, PF01159, PF01248, PF00411, PF00164, PF00416, PF00253, PF00833, PF00203, PF01090, PF00318, PF01282, PF03297,PF01283, PF01667, PF01015, PF00163, PF00333, PF00177, PF00410, PF01201, PF00380, PF08071)

\footnotetext{
${ }^{a}$ Shown are Pfam domains that were found among the top 500 most expressed isotigs in at least one library of each fungus and that are shared between all three fungi. In total 206 Pfam domains were found in this cohort (c.f. Figure 3B; Additional file 6). Pfam domains encoding protein motifs with unspecific or unknown functions are not shown. Bold indicates domains that were found among 25 expanded Pfam domains identified in the genome of $M$. haptotylum [20].
}

\section{Interspecific variation in gene expression}

To identify the variable sets of transcripts, that is, the transcripts that were differentially regulated depending on the fungal species, the expression levels of the putative orthologs identified using the UniRef50 clusters were compared among the libraries (Figure 5; Additional file 7). Among these were transcripts encoding peptidases (peptidase_M1 and peptidase_M24), lectins (FB_lectin, B_lectin, RicinB_lectin_2), tyrosinase, transcription factors, cell-signaling components, Atg8, various stress response proteins, proteins containing the WSC domain and the DUF3129 domain. DUF3129 is a domain of unknown function that is found in the gas1 protein of $M$. grisea, which participates in appressorial penetration and lesion formation [40]. Interestingly, DUF3129 was highly expressed in $M$. cionopagum but not expressed at all in A. dactyloides. DUF3129 was identified in 12 transcripts among the top 500 most expressed transcripts in the $\mathrm{Mc}(\mathrm{Hs})$ library. During $A$. oligospora infections, this domain was identified in one transcript in $\mathrm{Ao}(\mathrm{Mh})$ and in four transcripts in Ao 


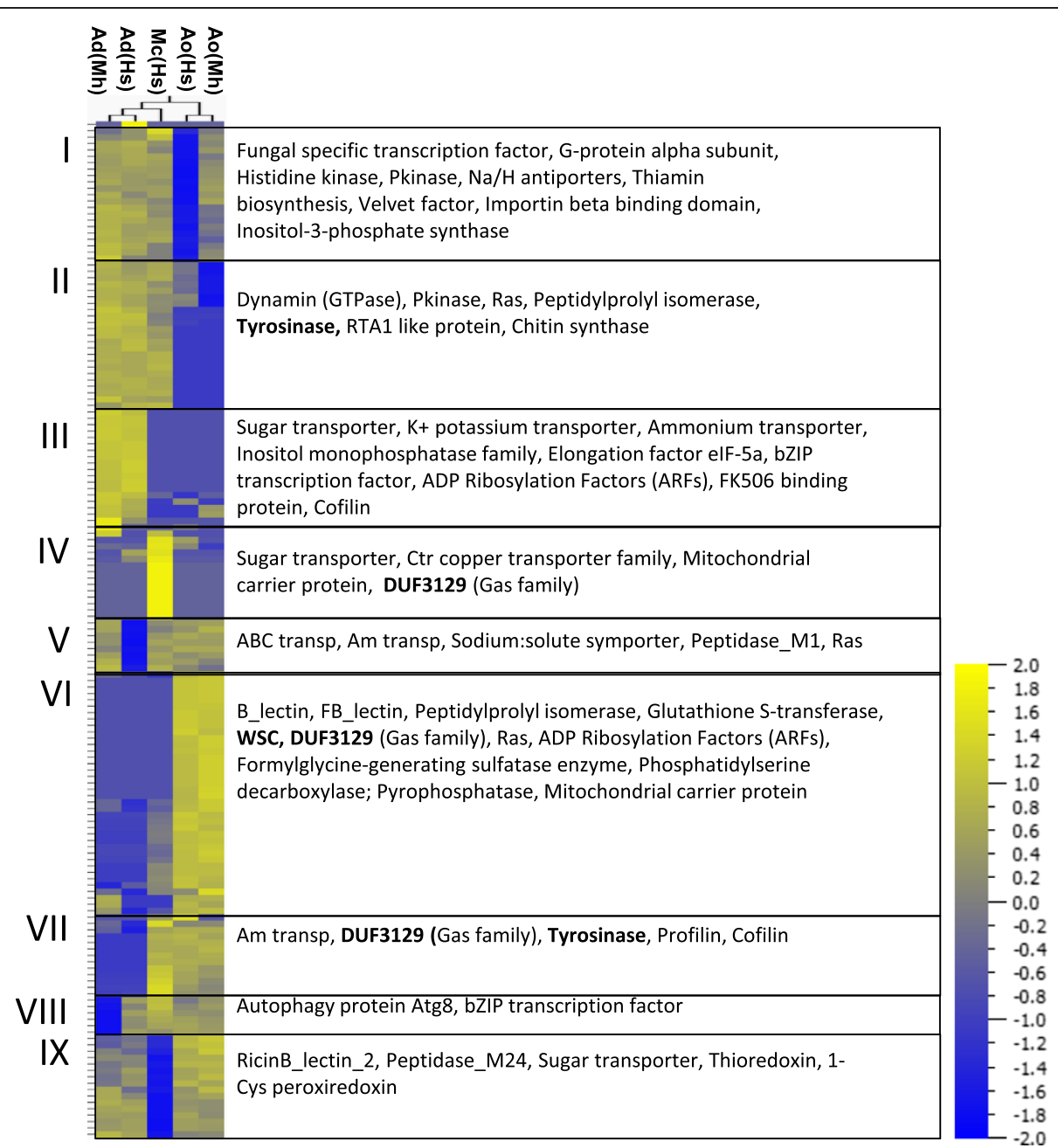

Figure $\mathbf{5}$ Heat map of gene expression levels of UniRef50 clusters. Gene expression levels of the $5 \%$ most highly expressed UniRef50 clusters passing a variance filtering of 0.3 (total 160) are shown. I to IX indicate nine clusters that were identified using hierarchical clustering of normalized, $\log _{2}$-transformed read counts (+1). The right panels shows annotations of the UniRef50 cluster sequences based on the presence of Pfam domains (Additional file 7). Bold indicates domains that were found among 25 expanded Pfam domains identified in the genome of $M$. haptotylum [20]. Ao(Mh) denotes A. oligospora and M. hapla; Ao(Hs), A. oligospora and H. schachtii; Ad(Mh), A. dactyloides and M. hapla; Ad(Hs), A. dactyloides and H. schachtii; and $\mathrm{Mc}(\mathrm{Hs}), \mathrm{M}$. cionopagum and $H$. schachtii.

(Hs) among the top 500 most expressed transcripts. DUF3129 is an expanded gene family in nematodetrapping fungi and both $M$. haptotylum and A. oligospora have 33 genes encoding this domain [20]. Seventeen of these genes were previously found among the $10 \%$ most expressed genes during nematode infection by $M$. haptotylum, whereas only two were among the $10 \%$ most expressed genes during nematode infection by A. oligospora [20]. The DUF3129 domain is thus highly expressed during infection among the species that form adhesive branches and adhesive knobs. Further studies are needed to investigate the function of the DUF3129 domain in the nematode-trapping fungi during infection.

Transcripts encoding the fungal fruit-body lectin (FB_lectin) and the D-mannose binding lectin (B_lectin) were only highly expressed in A. oligospora and not in the other two fungi (Figure 5). In the UniRef50 cluster containing the FB_lectin domain we identified the previously studied AOL lectin (Q00233) [41]. The transcript matching to this cluster was the third most expressed of all transcripts during $\mathrm{Ao}(\mathrm{Mh})$ infection and the 25th most expressed of all transcripts during $\mathrm{Ao}(\mathrm{Hs})$ infection. Earlier studies have shown that AOL functions as a storage protein during both saprophytic and parasitic growth [42]. However, deletion of this gene did not affect the fungus' ability to infect nematodes [43].

Previous studies have shown that proteins containing the carbohydrate-binding domain WSC comprise a large and rapidly evolving gene family in $M$. haptotylum [22]. Phylogenetic analysis of the $33 \mathrm{WSC}$-containing proteins in $M$. haptotylum revealed a clade of 15 WSC paralogs [22]. This clade contains only one (G1X6Q5) of the 16 
WSC proteins identified in A. oligospora [22]. Thirteen of the 15 WSC paralogs of $M$. haptotylum were at least twofold upregulated during the infection of the nematode C. briggsae [20]. In this study, transcripts encoding WSC proteins were highly expressed by all fungi during infection of plant-parasitic nematodes (Table 4). The largest number of transcripts encoding WSC domain proteins was expressed by $M$. cionopagum. In total, 19 transcripts of WSC domain proteins were identified in the $\mathrm{Mc}(\mathrm{Hs})$ library, of which seven were found among the top 500 transcripts. Four of these transcripts displayed closest sequence homology to proteins found in the expanded clade of WSC proteins of $M$. haptotylum [22]. The libraries of $A$. oligospora and $A$. dactyloides contained a lower number of transcripts of WSC domain proteins. Among the top 500 transcripts, the $\mathrm{Ao}(\mathrm{Hs}), \mathrm{Ao}(\mathrm{Mh})$ and $\mathrm{Ad}(\mathrm{Mh})$ libraries each had two WSC domain proteins, whereas none were found in the $\operatorname{Ad}(\mathrm{Hs})$ library. Transcripts displaying highest sequence homology to the previously mentioned A. oligospora protein G1X6Q5 were identified in both the $\mathrm{Ao}(\mathrm{Hs})$ and the $\mathrm{Ao}(\mathrm{Mh})$ libraries. The deduced proteins of two highly expressed WSC transcripts in $A$. dactyloides did not show any sequence similarity to the proteins found in the expanded clade of paralogs in M. haptotylum [22]. Taken together, the comparative transcriptome analysis shows that the WSC domain proteins comprise a large and divergent gene family that is highly expressed during pathogenesis in nematode-trapping fungi. The specific sets of genes that are expressed depend on the fungal species and the nematodes being infected, which suggests that the function of the WSC proteins is to contribute to the specialization of the trapping mechanisms.

\section{Virulence associated transcripts}

A BLAST search of the top 500 transcripts in each library was conducted in the pathogen-host interaction protein database (PHI-base) [44]. PHI-base contains experimentally verified pathogenicity, virulence and effector genes from fungi, oomycetes and bacterial pathogens. In total, 97 unique PHI-base genes were identified. Genes with sequence similarity to seven of them were found in at least ten gene models in either $M$. haptotylum or A. oligospora [20]. They included RBT4 from Candida albicans, which is necessary for virulence [45]. The function of RBT4 is unknown but it contains a CAP (Cysteine-rich secretory proteins, Antigen 5 and Pathogenesis-related 1 protein) domain [45]. Among the 97 identified PHI-base genes, 15 were highly expressed by all fungal species (Additional files 8 and 9) and 82 were highly expressed by one or two of the fungal species (Additional file 10). The PHI-base genes expressed by all fungi included stress response genes and several cell signaling genes containing the Ras domain. The PHI-base genes that differ in expression between the fungi included aspartic proteases and the gas 1 and gas 2 proteins of $M$. grisea [40] that contains the DUF3129 domain.

\section{Host-specific gene expression}

A scatter plot of the gene expression in A. oligospora during the infection of $M$. hapla versus $H$. schachtii showed that a majority of the genes had similar expression levels (Figure 6). However, 105 genes were expressed at levels at least 5-fold higher during infection of $M$. hapla than during infection of $H$. schachtii (Additional file 11), and 65 genes were expressed at levels at least 5-fold higher in $H$. schachtii than in M. hapla (Additional file 12). Genes predicted to encode secreted proteins were enriched among the differentially expressed genes in both nematodes. The proportion of secreted proteins among the upregulated genes in M. hapla (Ao(Mh) or $\mathrm{Ad}(\mathrm{Mh})$ ) was $12.4 \%$ (13 out of 105) and in H. schachtii (Ao(Hs) or $\mathrm{Ad}(\mathrm{Hs})$ ) was $13.8 \%$ (9 out of 65 ). In comparison, the proportion of secreted proteins among all genes that were used for the host-specific gene expression analysis was $7.3 \%$ (304 out of 4,138).

The differentially expressed genes encoding proteins with a predicted secretion signal were further characterized (Table 5). These included peptidases and several gene families that were expanded in the genomes of nematodetrapping fungi, such as DUF3129, WSC and tyrosinase [20]. One chitinase was identified that contains a LysM domain. Chitinases with LysM domains (CBM50) have been shown to have sequence similarity to the yeast killer toxin of Kluyveromyces lactis [46]. Five of the 22 secreted differentially expressed genes were assigned as SSPs with unknown function (Table 5).

\section{Conclusions}

This is the first study examining the variation in gene expression patterns among nematode-trapping fungi during infection of different host species. Comparative transcriptome analysis showed that the divergence in gene expression between the fungal species was significantly larger than that related to the nematode host. A core set of transcripts that were highly expressed by all three fungi was identified. This core set included subtilisins, aspartic proteases and proteins containing the CFEM domain. These genes were also highly expressed by A. oligospora and $M$. haptotylum during infection of C. briggsae [20]. Also within this core set, a putative nematotoxic protein was identified, the Ricin-B lectin. A more variable set of transcripts being regulated depending on the fungal species was also identified. A small set of genes was identified showing differential expression depending on the host. This set was enriched in genes encoding secreted proteins and also included several gene families that were expanded in the genomes of nematode-trapping fungi 


\section{A. oligospora $\quad \mathrm{r}=0.91$}



Figure 6 Host-specific gene expression in Arthrobotrys oligospora. Scatter plot of normalized mapped reads of A. oligospora and H. schachtii $(\mathrm{Ao}(\mathrm{Hs}))$ versus $A$. oligospora and $M$. hapla $(\mathrm{Ao}(\mathrm{Mh}))$. Genes that were regulated ( $\geq 1 \mathrm{read})$ in both libraries and that had $\geq 5$ reads in any of the libraries were included in the analysis, 4138 genes in total. The Pearson correlation coefficient ( $r$ ) of the comparison is also shown. The diagonal line $(y=x)$ shows transcripts with near identical expression levels. The dotted lines correspond to a five-fold expression difference.

[20]. Information on the genetic basis of the interspecific variation in the trapping mechanisms and host preferences fungi will be useful for researchers who are screening for more potent biological control agents of nematode-trapping fungi.

\section{Methods}

\section{Culture of organisms and infection experiments}

Cultures of A. oligospora (ATCC 24927), M. cionopagum (CBS 220.54) and A. dactyloides (CBS 109.37) were maintained on corn meal agar 1:10. Infested soil/roots of $M$. hapla (Strain E 226) were obtained from Prof. Dr. Gerrit Karssen (Plant Protection Service, HC Wageningen, the Netherlands) to start the culture of this nematode. Small pieces of roots infested with $M$. hapla were inoculated in rhizosphere of 3-week-old tomato plants raised in the green house of Department of Biology, Lund University, for induction of root knots. After 8 weeks, the infected tomato roots with well developed knots and egg masses were gently washed under running tap water. Egg masses of $M$. hapla were picked by fine forceps from knots of infected roots under a stereoscopic binocular microscope and surface disinfected for 1 minute in a $0.5 \% \mathrm{NaOCl}$ solution and rinsed three times in sterile distilled water. Egg masses were then collected in Petri dishes $(30 \mathrm{~mm})$ in sterile distilled water and incubated at $22 \pm 1^{\circ} \mathrm{C}$ for 48 hours for hatching of second-stage juveniles $\left(\mathrm{J}_{2}\right)$. After incubation, freshly hatched $\mathrm{J}_{2} \mathrm{~S}$ were separated from the egg masses and collated in Eppendorf tubes, surface sterilized with $0.5 \% \mathrm{NaOCl}$ for 2 minutes, and rinsed five times with sterilized distilled water, and used for infection experiments. $\mathrm{J}_{2} \mathrm{~s}$ of $\mathrm{H}$. schachtii were obtained from HZPC in the Netherlands (http://www.hzpc.com) and used for infection experiments after sterilization and washing as described for M. hapla.

Infection experiments were performed using a dialysis membrane assay [48]. Briefly, conidia of A. oligospora, $M$. cionopagum and $A$. dactyloides were inoculated onto several pieces of sterilized dialysis membrane (spectra/por 4, Spectrumlabs). The membranes were placed over plates containing modified low-nutrient mineral salt (LNM) medium $\left(\mathrm{KCl} 1.0 \mathrm{~g} / \mathrm{l}, \mathrm{MgSO}_{4} 0.2 \mathrm{~g} / \mathrm{l}\right.$, $\mathrm{ZnSO}_{4} .7 \mathrm{H}_{2} \mathrm{O} 0.88 \mathrm{mg} / \mathrm{l}, \mathrm{FeCl}_{3} \cdot 6 \mathrm{H}_{2} \mathrm{O} 3.0 \mathrm{mg} / \mathrm{l}$, thiamine$\mathrm{HCl} 0.2 \mathrm{mg} / \mathrm{l}$, biotin $0.005 \mathrm{mg} / \mathrm{l}$, L-phenylalanine-L-valine $0.1 \mathrm{~g} / \mathrm{l}$, agar $10 \mathrm{~g} / \mathrm{l}, \mathrm{pH}$ 6.5) [48,49]. Infection structures (traps) were induced by adding 40-50 specimens of the nematode Panagrellus redivivus L. (Goodey) to the hyphae growing on each dialysis membrane. $P$. redivivus was grown axenically in a soya peptone-liver extract [50]. After several days, when substantial amount of traps have been developed and all added nematodes have been killed and digested, the infection experiments were started by adding 75-100 surface sterilized second-stage juveniles of M. hapla or $H$. schachtii. The following five combinations of fungi and nematodes were examined: A. oligospora and $M$. hapla (designated Ao(Mh)), A. oligospora and H. schachtii (Ao(Hs)), A. dactyloides and M. hapla (Ad (Mh)), A. dactyloides and H. schachtii (Ad(Hs)), and $M$. cionopagum and $H$. schachtii $(\mathrm{Mc}(\mathrm{Hs}))$. The infection was followed under a light microscope, and the number of 
Table 5 Differentially expressed genes encoding secreted proteins in A. oligospora during infection of M. hapla or H. schachtii ${ }^{a}$

\begin{tabular}{|c|c|c|c|c|}
\hline UniProt ID & Description $^{\mathbf{b}}$ & Pfam & Length $^{c}$ & Fold change \\
\hline \multicolumn{5}{|c|}{ Upregulated in M. hapla } \\
\hline G1X4P0 & Histidine acid phosphatase & His_Phos_2 & 476 & 9.1 \\
\hline G1XTC9 & Patched sphingolipid transporter & Patched & 1292 & 9.1 \\
\hline G1XF88 & Uncharacterized protein & - & $251^{e}$ & 7.7 \\
\hline G1XGH3 & IgE-binding protein & - & 195 & 7.4 \\
\hline G1XHE8 $^{d}$ & - & - & $196^{\mathrm{e}}$ & 6.9 \\
\hline G1X1U4 & Glucosidase 2 subunit beta & - & 553 & 6.3 \\
\hline G1X8R3 & GPI anchored cell-wall protein & But2 & 328 & 6.2 \\
\hline G1XCM7 & Beta-galactosidase & Glyco_hydro_35, BetaGal_dom2, BetaGal_dom3, BetaGal_dom4_5 & 982 & 5.7 \\
\hline G1XET4 & Peptidase S41 & Peptidase_S41 & 702 & 5.7 \\
\hline G1XGl5 & Uncharacterized protein & - & $107^{\mathrm{e}}$ & 5.3 \\
\hline G1XPV3 & Uncharacterized protein & - & $173^{e}$ & 5.3 \\
\hline G1XJP0 & Uncharacterized protein & - & 606 & 5.1 \\
\hline G1XLE5 & Aminopeptidase $Y$ & PA, Peptidase_M28 & 503 & 5.1 \\
\hline \multicolumn{5}{|c|}{ Upregulated in H. schachtii } \\
\hline $\mathrm{G} 1 \times 7 \mathrm{Q} 6^{\mathrm{d}}$ & - & - & 300 & 15.8 \\
\hline G1XQA9 & Uncharacterized protein & - & 707 & 15.8 \\
\hline G1XU57 & $\mathrm{ABC}$ transporter & ABC_tran, ABC2_membrane & 1047 & 15.8 \\
\hline $\mathrm{G} 1 \times 110^{d}$ & - & - & $151^{e}$ & 11.4 \\
\hline G1XR64 & Uncharacterized protein & DUF3129 & 656 & 10.5 \\
\hline G1XF27 & WSC-domain-containing protein & WSC & 625 & 9.6 \\
\hline G1XEV7 & Chitinase & Glyco_hydro_18, LysM & 1232 & 8.8 \\
\hline G1XM84 & Tyrosinase & Tyrosinase & 387 & 8.8 \\
\hline G1XC55 & Uncharacterized protein & DUF3129 & 454 & 6.4 \\
\hline
\end{tabular}

${ }^{\mathrm{a}}$ Genes encoding proteins with a predicted secretion signal that were upregulated at least 5-fold in the sample A. oligospora infecting M. hapla (Ao(Mh)) compared to the sample $A$. oligospora infecting $H$. schachtii $(\mathrm{Ao}(\mathrm{Hs}))$ and in $\mathrm{Ao}(\mathrm{Hs})$ compared to $\mathrm{Ao}(\mathrm{Mh})$. Genes that were expressed $(\geq 1$ read) in both libraries and that had $\geq 5$ read in any of the libraries were included in the analysis, in total 4138 genes.

${ }^{b}$ Non-annotated genes of $A$. oligospora were further characterized by searches through the UniProt database [47] using the BLASTP algorithm [25] with an E-value threshold of 1e-10. Fungal sequences were chosen among the top hits.

'The length of the protein sequence in aa.

dOrphan, protein that lacks known homologs and does not contain any Pfam domains.

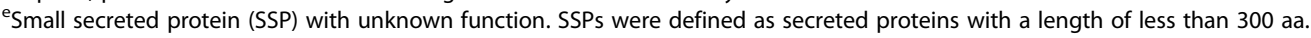

trapped, paralyzed (i.e. with arrested movements), and colonized (hyphae growing inside the capture nematode) were counted after various time periods. For each fungal and nematode interaction, 10 replications were used. Dialysis membranes having fungal and nematode interaction of each stage were quickly transferred into liquid nitrogen and ground. Materials were collected from all infection stages (trapped, paralyzed and infected (colonized) nematodes). The ground material was stored at $-80^{\circ} \mathrm{C}$ until use.

\section{RNA extraction, CDNA library construction and sequencing}

Total RNA was extracted from each infection stage using the RNeasy Plant Mini kit and the RLC buffer (Qiagen) and subsequently quantified using a NanoDrop
2000C spectrophotometer (Thermo Scientific). RNA integrity was inspected using a RNA 6000 Pico kit on a 2100 BioAnalyzer (Agilent). Approximately equal amounts of RNA from the three infection stages of each fungusnematode combination were pooled. The RNA pools were concentrated by precipitation using ammonium acetate/ glycogen/ethanol as described in the MicroPoly(A)Purist Kit manual (Ambion).

From total RNA, mRNA was isolated using the PolyATtract kit (Promega) according to manufacturer's instructions. Double-stranded cDNA was synthesized using the cDNA Synthesis System (Roche Diagnostics) according to the GS FLX Titanium cDNA Rapid Library preparation protocol (454/Roche) and using adaptors with Multiplex Identifiers (MIDs) that allow for pooling of the libraries prior sequencing. Library concentration was 
assessed by qPCR on a Mx3005P instrument (Stratagene) and using the KAPA Library Quantification Kit - 454 Titanium (Lib-L)/Universal (Kapa Biosystems). Based on the qPCR results all libraries were pooled to contain an equal molar amount of each library. Titration and library production (aiming at $7-16 \%$ enrichment) was performed using emulsion PCR and the Lib-L kit (454/Roche). DNA-containing beads were enriched and counted using a CASY Cell Counter DT (Roche Innovatis AG), processed using aXLR70 sequencing kit (454 Life Sciences/Roche Diagnostics), and loaded onto a picotiter plate for pyrosequencing on a 454 Life Sciences Genome Sequencer FLX machine (454 Life Sciences/Roche Diagnostics). Sequencing was conducted at the Lund University Sequencing Facility (Faculty of Science).

\section{Bioinformatic analyses}

The reads obtained from the 454 sequencing were filtered, assembled and analysed according to the flowchart shown in Figure 2. Reads matching rRNA were removed using the BLASTN algorithm [25] with an E-value threshold of $1 \mathrm{e}-5$ against a custom made database of rRNA sequences obtained from the $5 \mathrm{~S}$ rRNA database [51] and the SILVA rRNA database [52]. The remaining reads for each of the five libraries were assembled separately using the GS de novo assembler 2.6 (454 Life Sciences/Roche Diagnostics) with the -cdna option. The reads were assembled into 17785 isotigs and 10 contigs with a length $>500 \mathrm{bp}$. In the following text, both categories were referred as isotigs, i.e. transcripts. Low abundance isotigs with less than five reads and isotigs with a length shorter than $100 \mathrm{bp}$ were removed. Isotigs with top hits to non-fungal species in the UniProt database [47] (the BLASTX search) [25] were also removed. The filtered dataset contained 17446 isotigs.

The filtered isotigs from the two A. oligospora samples were mapped to the A. oligospora genome using Gmap [53] to assess the quality of the de novo isotig assemblies. In total, 3944 out of 3952 isotigs (including 2634 istotigs from the $\mathrm{Ao}(\mathrm{Mh})$ library and 1318 isotigs from the Ao (Hs) library, Table 2)) matched the genome indicating efficient filtering of non-fungal transcripts. Only 75 of the 3944 isotigs aligned to more than one position in the genome giving a total of 4019 genome regions aligning to the isotigs. The low number of isotigs with multiple matches indicates a low frequency of chimeric transcripts. To further investigate the quality of the transcriptome assembly, we compared the genome sequences of isotig alignments with the 11479 predicted genes from the A. oligospora genome using the Eval software [54]. All of the 4019 genome regions matched to the predicted $A$. oligospora genes. In total, 2652 A. oligospora genes were matched giving on average 1.5 isotigs per predicted gene which suggest that some of the isotigs may represent alternative splicing forms. In total, 94.6 percent of the aligned genome regions (3 803 out of 4 019) contained both start and stop codon (i.e. considered complete by the Eval software). The mean length of the isotigs was 1 109 basepairs compared to 1498 basepairs for the $A$. oligospora gene models. The difference in length may at least partly be due to alternative splicing forms where exon skipping will give shorter transcripts than the predicted gene models. The high proportion of successful matches to the A. oligospora genome as well as to its genes, the large number of complete isotigs and the long mean isotig length clearly indicate that most of the filtered isotigs have been correctly assembled into near full length transcripts.

The filtered isotigs were used to generate two different data sets (Figure 2). The first data set ("Highly expressed transcripts") was normalized using two different approaches; reads per kilobase pair $(\mathrm{kb})$, (the number of aligned reads per transcript was divided by the transcript length), and the reads per kilobase per million reads (RPKM) method [55] (Additional file 1). The isotigs were annotated based on homology using the BLASTX algorithm [25] (threshold values of 1e-10) to the UniProt sequence database [47] and proteins of $M$. haptotylum [20]. The isotigs were also annotated using the pfam_scan.pl tool (ftp://ftp.sanger.ac.uk/ pub/databases/Pfam/Tools/) to search the Pfam-A family protein database [56] with default thresholds. Secretion signals were predicted using the SignalP 4.0 algorithm [57]. Isotigs were considered to encode putative secreted proteins if fulfilling at least one of the following three criteria: 1) Isotigs having a secretion signal in the same frame as the Pfam domain; 2) Isotigs having a secretion signal in the longest predicted open-reading frame (ORF) in the same frame as the BLASTX match (threshold value of 1e-10) to a protein in the UniProt database or protein from $M$. haptotylum; 3) Isotigs having a BLASTX match to a protein in the UniProt database or protein from $M$. haptotylum that contains a secretion signal. Orphans were identified as isotigs lacking both Pfam domains and BLASTX matches in the UniProt database and the $M$. haptotylum genome (threshold value of 1e-5) against a species other than itself. Orphans with a secretion signal in the longest ORF were considered to be putative secreted proteins. PCA and hierarchical clustering were performed using the Omics Explorer ver. 2.2 (Qlucore). Virulence-related genes were identified by BLASTX [25] similarity searches against the PHI-base database version 3.2 [44] using a cutoff of $<1 \mathrm{e}-10$.

The second data set ("Differentially expressed UniRef50 clusters") was obtained by matching the isotig sequences to UniRef50 clusters [26]. The UniRef50 clusters contain a representative of UniProt sequences that show 50\% sequence similarity and $80 \%$ overlap with the longest sequence in the cluster. The isotigs with the highest 
BLASTX score to a UniRef50 sequence cluster from each library were considered as putative orthologs giving maximum one isotig from each species for any given UniRef50 cluster to take alternative splicing into account. Sometimes no isotig from one species has a significant match to a particular UniRef50 cluster (cutoff 1e-10). The transcript abundance of these putative orthologs, called "UniRef50 clusters" was normalized to correct for different library sizes using the R/Bioconductor software package DESeq 1.10.1 [27] (Additional file 2).

To identify the third data set, "Host-specific gene expression" (Figure 2), GSMapper 2.8 (454 Life Sciences/Roche Diagnostics) was used with the -cdna and -cref parameters to map the reads from the two $A$. oligospora libraries $(\mathrm{Ao}(\mathrm{Mh})$ and $\mathrm{Ao}(\mathrm{Hs})$ ) against the coding sequences of the 11479 genes predicted in the A. oligospora genome [19]. The read counts were normalized using DESeq [27]. A homology search of the mapped A. oligospora proteins was carried out using the BLASTP algorithm [25] to the UniProt database and proteins of $M$. haptotylum (threshold values of 1e-5). Secretory A. oligospora proteins were predicted using SignalP 4.0 [57].

\section{Sequence accession numbers}

Sequences can be accessed from the database http://mbioserv2.mbioekol.lu.se/NematodeTrappingFungi/. The short read pyrosequences from $\mathrm{Ad}(\mathrm{Mh}), \mathrm{Ad}(\mathrm{Hs}), \mathrm{Ao}(\mathrm{Mh}), \mathrm{Ao}(\mathrm{Hs})$ and $\mathrm{Mc}(\mathrm{Hs})$ are available at NCBI SRA database with the Bioproject IDs PRJNA230433, PRJNA230458, PRJNA230459, PRJNA230446 and PRJNA230448, respectively.

\section{Additional files}

Additional file 1: Evaluation of procedures used for normalizing sequence reads.

Additional file 2: Evaluations of procedures used for normalizing gene expression levels of UniRef50 clusters.

Additional file 3: Summary of the annotation of the $\mathbf{5 0 0}$ most expressed transcripts.

Additional file 4: Annotation of the 500 most expressed transcripts. Additional file 5: PCA analysis of highly expressed UniRef50 clusters.

Additional file 6: Number of pfam domains expressed by all fungi during nematode infection.

Additional file 7: Isotigs and Pfam annotations of UniRef50 clusters shown in the heatmap of Figure 5.

Additional file 8: Isotigs displaying sequence similarity to proteins in the PHI-database.

Additional file 9: Virulence associated proteins highly expressed by all fungi.

Additional file 10: Variations in expression of virulence associated proteins.

Additional file 11: Upregulated genes in A. oligospora during infection of $M$. hapla as compared with $H$. schachtii.

Additional file 12: Upregulated genes in A. oligospora during infection of $H$. schachtii as compared with M. hapla.
Competing interests

The authors declare that they have no competing interests.

\section{Authors' contributions}

Conceived and designed the experiments: AT, DK Performed the experiments: DK, EF cDNA library preparations and transcriptome sequencing: EF. Analyzed the data: K-MA, JB, DA, AT Wrote the paper: K-MA with input from AT, DA, JB and DK. All authors read and approved the final manuscript.

\section{Acknowledgements}

This work was supported by grants from the Swedish Research Council (VR) DK was supported by a DST (Department of Science and Technology, Gov. of India) BOYSCAST fellowship.

\section{Author details}

1Department of Biology, Microbial Ecology Group, Lund University, Ecology Building, 22362 Lund, Sweden. Department of Genetics and Plant Breeding, College of Agriculture, Narendra Deva University of Agriculture and Technology, Kumarganj, Faizabad 224229, Uttar Pradesh (U.P.), India. ${ }^{3}$ Department of Biology, BILS Bioinformatics Infrastructure for Life Sciences, Lund University, Ecology Building, 22362 Lund, Sweden.

Received: 12 February 2014 Accepted: 24 September 2014

Published: 11 November 2014

\section{References}

1. Barron GL: The nematode-destroying fungi. Guelph: Canadian Biological Publications; 1977

2. Tunlid A, Ahrén D: Molecular mechanisms of the interaction between nematode-trapping fungi and nematodes: Lessons from genomics. In Biological control of plant-parasitic nematodes, vol 11. Edited by Davies K, Spiegel Y. Springer: Netherlands; 2011:145-169.

3. Dijksterhuis J, Veenhuis M, Harder W, Nordbring-Hertz B: Nematophagous fungi: physiological aspects and structure-function relationships. Adv Microb Physiol 1994, 36:111-143.

4. Nordbring-Hertz B, Jansson HB, Tunlid A: Nematophagous Fungi. John Wiley \& Sons, Chichester: In Encyclopedia of Life Sciences; 2011.

5. Liou GY, Tzean SS: Phylogeny of the genus Arthrobotrys and allied nematode-trapping fungi based on rDNA sequences. Mycologia 1997, 89:876-884.

6. Ahrén D, Ursing BM, Tunlid A: Phylogeny of nematode-trapping fungi based on 18S rDNA sequences. FEMS Microbiol Lett 1998, 158:179-184.

7. Li Y, Hyde KD, Jeewon R, Cai L, Vijaykrishna D, Zhang KQ: Phylogenetics and evolution of nematode-trapping fungi (Orbiliales) estimated from nuclear and protein coding genes. Mycologia 2005, 97:1034-1046.

8. Yang Y, Yang E, An ZQ, Liu XZ: Evolution of nematode-trapping cells of predatory fungi of the Orbiliaceae based on evidence from rRNA-encoding DNA and multiprotein sequences. Proc Natl Acad SCl U S A 2007, 104:8379-8384.

9. Higgins ML, Pramer D: Fungal Morphogenesis - Ring formation and closure by Arthrobotrys dactyloides. Science 1967, 155:345-346.

10. Heintz CE, Pramer D: Ultrastructure of nematode-trapping fungi. J Bacteriol 1972, 110:1163-1170.

11. Liu KK, Tian JQ, Xiang MC, Liu XZ: How carnivorous fungi use three-celled constricting rings to trap nematodes. Protein Cell 2012, 3:325-328.

12. Tunlid A, Jansson HB, Nordbring-Hertz B: Fungal attachment to nematodes. Mycol Res 1992, 96:401-412.

13. Nordbring-Hertz B, Jansson HB, Friman E, Persson $Y$, Dackman C, Trude H, Poloczek E, Feldman R: Nematophagous fungi. Göttingen, Germany: Institut für den Wissenschaftlichen Film; 1995. Film No. C 1851.

14. Jansson HB, Nordbring-Hertz B: Interactions between nematophagous fungi and plant-parasitic nematodes - Attraction, induction of trap formation and capture. Nematologica 1980, 26:383-389.

15. Rosenzweig WD, Premachandran D, Pramer D: Role of trap lectins in the specificity of nematode capture by fungi. Can J Microbiol 1985, 31:693-695.

16. Singh KP, Jaiswal RK, Kumar N, Kumar D: Nematophagous fungi associated with root galls of rice caused by Meloidogyne graminicola and its control by Arthrobotrys dactyloides and Dactylaria brochopaga. J Phytopathol 2007, 155:193-197. 
17. Kumar D, Singh KP: Assessment of predacity and efficacy of Arthrobotrys dactyloides for biological control of root knot disease of tomato. J Phytopathol 2006, 154:1-5.

18. Jaffee BA, Muldoon AE: Susceptibility of root-knot and cyst nematodes to the nematode-trapping fungi Monacrosporium ellipsosporum and $M$. cionopagum. Soil Biol Biochem 1995, 27:1083-1090.

19. Yang J, Wang L, Ji X, Feng Y, Li X, Zou C, Xu J, Ren Y, Mi Q, Wu J, Liu S, Liu Y, Huang X, Wang H, Niu X, Li J, Liang L, Luo Y, Ji K, Zhou W, Yu Z, Li G, Liu Y, Li L, Qiao M, Feng L, Zhang KQ: Genomic and proteomic analyses of the fungus Arthrobotrys oligospora provide insights into nematode-trap formation. PLoS Pathog 2011, 7:e1002179.

20. Meerupati T, Andersson KM, Friman E, Kumar D, Tunlid A, Ahrén D: Genomic mechanisms accounting for the adaption to parasitism in nematode-trapping fungi. PLoS Genet 2013, 9:e1003909.

21. Tautz D, Domazet-Loso T: The evolutionary origin of orphan genes. Nat Rev Genet 2011, 12:692-702.

22. Andersson KM, Meerupati T, Levander F, Friman E, Ahrén D, Tunlid A: Proteome of the nematode-trapping cells of the fungus Monacrosporium haptotylum. Appl Environ Microbiol 2013, 79:4993-5004.

23. Williamson VM, Gleason CA: Plant-nematode interactions. Curr Opin Plant 2003, 6:327-333.

24. Bird $\mathrm{A}$ : The development and organization of skeletal structures in nematodes. In Croll NA: The organization of nematodes. London: Academic Press; 1976:107-137.

25. Camacho C, Coulouris G, Avagyan V, Ma N, Papadopoulos J, Bealer K, Madden TL: BLAST plus: architecture and applications. BMC Bioinformatics 2009, 10:421.

26. Suzek BE, Huang HZ, McGarvey P, Mazumder R, Wu CH: UniRef: comprehensive and non-redundant UniProt reference clusters. Bioinformatics 2007, 23:1282-1288.

27. Anders $S$, Huber W: Differential expression analysis for sequence count data. Genome Biol 2010, 11:R106.

28. Kulkarni RD, Kelkar HS, Dean RA: An eight-cysteine-containing CFEM domain unique to a group of fungal membrane proteins. Trends Biochem Sci 2003, 28:118-121.

29. Nakatogawa $H$, Ichimura $Y$, Ohsumi Y: Atg8, a ubiquitin-like protein required for autophagosome formation, mediates membrane tethering and hemifusion. Cell 2007, 130:165-178

30. Chen YL, Gao Y, Zhang KQ, Zou CG: Autophagy is required for trap formation in the nematode-trapping fungus Arthrobotrys oligospora. Environ Microbiol Rep 2013, 5:511-517.

31. Endo $Y$, Tsurugi K: RNA N-Glycosidase activity of ricin A-chain - Mechanism of action of the toxic lectin ricin on eukaryotic ribosomes. J Biol Chem 1987, 262:8128-8130.

32. Michiels K, Van Damme EJM, Smagghe G: Plant-insect interactions: What can we learn from plant lectins? Arch Insect Biochem Physiol 2010, 73:193-212.

33. Wohlschlager T, Butschi A, Zurfluh $K$, Vonesch SC, Keller UAD, Gehrig P Bleuler-Martinez S, Hengartner MO, Aebi M, Kunzler M: Nematotoxicity of Marasmius oreades agglutinin (MOA) depends on glycolipid binding and cysteine protease activity. J Biol Chem 2011, 286:30337-30343.

34. Hamshou M, Smagghe G, Shahidi-Noghabi S, De Geyter E, Lannoo N, Van Damme EJM: Insecticidal properties of Sclerotinia sclerotiorum agglutinin and its interaction with insect tissues and cells. Insect Biochem Mol Biol 2010, 40:883-890.

35. Tunlid A, Jansson S: Proteases and their involvement in the infection and immobilization of nematodes by the nematophagous fungus Arthrobotrys oligospora. Appl Environ Microbiol 1991, 57:2868-2872.

36. Åhman J, Johansson T, Olsson M, Punt PJ, van den Hondel CA, Tunlid A: Improving the pathogenicity of a nematode-trapping fungus by genetic engineering of a subtilisin with nematotoxic activity. Appl Environ Microbiol 2002, 68:3408-3415.

37. Morano KA, Grant CM, Moye-Rowley WS: The response to heat shock and oxidative stress in Saccharomyces cerevisiae. Genetics 2012, 190:1157-1195.

38. Heller J, Tudzynski P: Reactive oxygen species in phytopathogenic fungi: Signaling, development, and disease. Annu Rev Phytopathol 2011, 49:369-390

39. Egan MJ, Wang ZY, Jones MA, Smirnoff N, Talbot NJ: Generation of reactive oxygen species by fungal NADPH oxidases is required for rice blast disease. Proc Natl Acad Sci U S A 2007, 104:11772-11777.
40. Xue CY, Park G, Choi WB, Zheng L, Dean RA, Xu JR: Two novel fungal virulence genes specifically expressed in appressoria of the rice blast fungus. Plant Cell 2002, 14:2107-2119.

41. Rosén S, Ek B, Rask L, Tunlid A: Purification and characterization of a surface lectin from the nematode-trapping fungus Arthrobotrys oligospora. J Gen Microbiol 1992, 138:2663-2672.

42. Rosén S, Sjollema K, Veenhuis M, Tunlid A: A cytoplasmic lectin produced by the fungus Arthrobotrys oligospora functions as a storage protein during saprophytic and parasitic growth. Microbiology 1997, 143:2593-2604.

43. Balogh J, Tunlid A, Rosén S: Deletion of a lectin gene does not affect the phenotype of the nematode-trapping fungus Arthrobotrys oligospora. Fungal Genet Biol 2003, 39:128-135.

44. Winnenburg R, Urban M, Beacham A, Baldwin TK, Holland S, Lindeberg M, Hansen H, Rawlings C, Hammond-Kosack KE, Kohler J: PHI-base update: additions to the pathogen-host interaction database. Nucleic Acids Res 2008, 36:572-576.

45. Braun BR, Head WS, Wang MX, Johnson AD: Identification and characterization of TUP1-regulated genes in Candida albicans. Genetics 2000, 156:31-44.

46. Tzelepis GD, Melin P, Jensen DF, Stenlid J, Karlsson M: Functional analysis of glycoside hydrolase family 18 and 20 genes in Neurospora crassa. Fungal Genet Biol 2012, 49:717-730.

47. Bairoch A, Apweiler R, Wu CH, Barker WC, Boeckmann B, Ferro S, Gasteiger E, Huang H, Lopez R, Magrane M, Martin MJ, Natale DA, O'Donovan C, Redaschi N, Yeh LS: UniProt: the Universal Protein knowledgebase. Nucleic Acids Res 2004, 32:115-119.

48. Nordbring-Hertz B: Dialysis membrane technique for studying microbial interaction. Appl Environ Microbiol 1983, 45:290-293.

49. Nordbring-Hertz B: Peptide-induced morphogenesis in the nematode-trapping fungus Arthrobotrys oligospora. Physiol Plant 1973, 29:223-233.

50. Nordbring-Hertz B: Nematode-induced morphogenesis in predacious fungus Arthrobotrys oligospora. Nematologica 1977, 23:443-451.

51. Szymanski M, Barciszewska MZ, Erdmann VA, Barciszewski J: 5 S ribosomal RNA database. Nucleic Acids Res 2002, 30:176-178.

52. Quast C, Pruesse E, Yilmaz P, Gerken J, Schweer T, Yarza P, Peplies J, Glockner FO: The SILVA ribosomal RNA gene database project: improved data processing and web-based tools. Nucleic Acids Res 2013, 41:D590-D596.

53. Wu TD, Watanabe CK: GMAP: a genomic mapping and alignment program for mRNA and EST sequences. Bioinformatics 2005, 21:1859-1875.

54. Keibler E, Brent M: Eval: a software package for analysis of genome annotations. BMC Bioinformatics 2003, 4:50.

55. Mortazavi A, Williams BA, Mccue K, Schaeffer L, Wold B: Mapping and quantifying mammalian transcriptomes by RNA-Seq. Nat Methods 2008, 5:621-628.

56. Punta $M$, Coggill $P C$, Eberhardt RY, Mistry J, Tate J, Boursnell C, Pang N Forslund K, Ceric G, Clements J, Heger A, Holm L, Sonnhammer EL, Eddy SR, Bateman A, Finn RD: The Pfam protein families database. Nucleic Acids Res 2012, 40:D290-D301.

57. Petersen TN, Brunak S, von Heijne G, Nielsen H: SignalP 4.0: discriminating signal peptides from transmembrane regions. Nat Methods 2011, 8:785-786.

doi:10.1186/1471-2164-15-968

Cite this article as: Andersson et al:: Interspecific and host-related gene expression patterns in nematode-trapping fungi. BMC Genomics 2014 15:968. 